\title{
Predictive Analysis of Postpartum Haemorrhage Using Deep Learning Technique
}

\author{
S.V.Ruphitha ${ }^{\mathrm{a}, 1}$ and Dr.V.D.Ambeth $\mathrm{Kumar}^{\mathrm{b}}$ \\ a PG Scholar, Dept of CSE, Panimalar Engineering College, Chennai, India \\ ${ }^{\mathrm{b}}$ Professor, Dept of CSE, Panimalar Engineering College, Chennai, India
}

\begin{abstract}
Postpartum haemorrhage is the prime source of parental fatality. Postpartum haemorrhage occurs extra blood loss after delivery. If the blood loss occurs more than $500 \mathrm{ml}$, it may create a problem in blood pressure where the women has to go through a lot of pain. Presently, the complete cure are still under research and there is no result which helps to dwindle the endanger of postpartum haemorrhage. Intent process is so supportive inclassifying the danger circumstances and current automation is used. The methodology used here is Deep Learning technique which will be easier to conclude the postpartum haemorrhage in the previous phase. There is an certain stage of postpartum haemorrhage where it can control the blood loss and save the women by permitting higher level treatments. Haemorrhage is one of the major factor responsible for maternal death. Haemorrhage may occur before, during or after delivery of placenta. Based on the amount of blood flow, postpartum haemorrhage will be classified in to two types such as primary and secondary pph. To manage all these problems, methods are handled based on respective situation.
\end{abstract}

Keywords: Women, Risk factors, Postpartum Haemorrhage, Deep Learning Technique.

\section{Introduction}

Postpartum Bleeding is one of the major cause of parental fatality accounting for $60 \%$ of maternal deaths in developing countries. Statistics states that the death rate of women is peak within the 4 hours of delivery. $75 \%$ cases were managed by medical methods whereas rest of the cases are managed by surgical methods. Uterine atony were the highest significant source of postpartum haemorrhage. Postpartum Haemorrhage can be identified by the risk factors such as temperature, blood pressure, sweat rate, heart rate. After maternity period, the pregnant women may attain postpartum haemorrhage which may be primary or secondary pph. Basically, postpartum haemorrhage is divided into two parts such as primary postpartum haemorrhage which occurs within 24 hours of delivery and secondary postpartum haemorrhage which is said to be an retained endometritis.

${ }^{1}$ S.V.Ruphitha, PG Scholar, Dept of CSE, Panimalar Engineering College, Chennai, India E-mail:ruphitha1798@gmail.com 
For the above issues, a new proposed method which is known as deep learning technique will be introduced. The proposed method is to analyse the parameters of women those who suffer the most in labour ward during postpartum haemorrhage. The proposed classification methods have high accuracy to predict the postpartum haemorrhage patients and it also has a fast training process, so it is easy to predict whether the patients are affected by postpartum haemorrhage or not.

\section{Related work}

Identification of Postpartum Infection Type using Mamdani Fuzzy System by Salamah, $\mathrm{U}$ [1] a different and un noticed risk factor that is VON WILLIBRAND disease is mentioned and the crucial to management of PPH is early acceptance and treatment. Administration of PPH differs for both the case that includes primary and secondary (i.e)intrauterine massage, oxytocin infusion, methyl prostaglandin and hysterectomy respectively. Postpartum Haemorrhage: A Recurring Pregnancy Complication by Michelle Kominiarek,A [2] provides three methods (i.e) Mechanical: uterine massage, Pharmacological : uterotonics in varying combinations. Surgical: Balloon tamponade, B- lynch sutures, hysterectomy. All possibilities and risk factors are explained and the respective solutions to given is mentioned both through medicines and surgeries. A proposal for postpartum support based on natural language generation model by Zeni Montenegro, J. L [3] tells about the usage of misoprostol and improvement in the third stage of labour. Performing surgeries that include B- lynch sutures, hysterectomy is performed to overcome Uterine atony, cervical damage, retained placenta. Postpartum Haemorrhage by Gowri Ramanathan [4] discusses the major issues addressed and also the Usage of first line therapies and if incurable then adopted to second line therapies and in worst case all advanced surgeries are performed according to the complication. Management of Postpartum Haemorrhage by Rancogz [5] provides the case study and the Concept of 4T (Tissue, Thrombin, Tone, Trauma), uterine atony, resuscitation, Uterine massage, Uterotonic Agent. Cloud-Based Multinomial Logistic Regression for Analysing Maternal Mortality Data in Postpartum Period by Radite Purwahana, R [6] discusses about the emerging value of misoprostol and global initiatives in the management of $\mathrm{PPH}$, will reduce the incidence of this life-threatening condition in the next few years[21-29]. A condom uterine balloon device among referral facilities in Dar Es Salaam: An assessment of perceptions, barriers and facilitators one year after implementation by Adegoke, $\mathrm{O}$ [7] gives a successful deliveries with very less inconvenience and damage of the genital parts of women. Very much useful in easy movement of baby in the vaginal canal without tears in the vagina, cervix. Diagnosis and management of postpartum haemorrhage." Diagnosis and management of postpartum haemorrhage by Edwin Chandraharan [8] addresses an issue on Vaginal tears, cervical damage under macrosomy conditions[15-20].

\section{System Architecture}

The below fig. 1 represents the complete process of the proposed system. Where the first thing is to get the patient's details and to do data pre-processing. The database will be cleaned once the pre-processing method is done. Then training and testing is to be done by collecting the data and this will be done with the three important steps such as 
Confusion matrix, Activation layer and Performance metrices. Confusion matrices will be calculated in the form of true positive, true negative, false positive and false negative. Activation layer consists of relu, tanh, sigmoid. The performance will be measured by using various steps. By applying deep learning techniques, it will be easier to conclude the postpartum haemorrhage at an previous stage. The results will be collected for the two techniques which is used under the concept of convolutional neural network and it will be compared to predict postpartum haemorrhage.

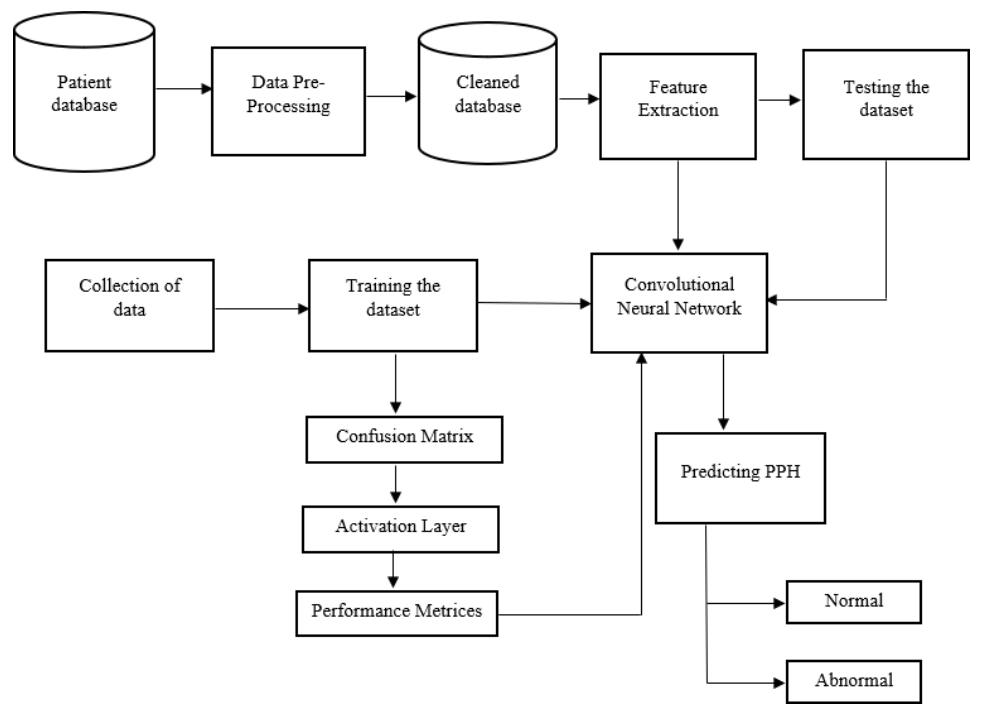

Figure 1. Identifying Postpartum Haemorrhage using Deep LearningTechnique

\section{Dataflow Diagrams}

\subsection{Level 0}

Collect a datasets from health care. Process the collected dataset and findingpostpartum haemorrhage affected patients.

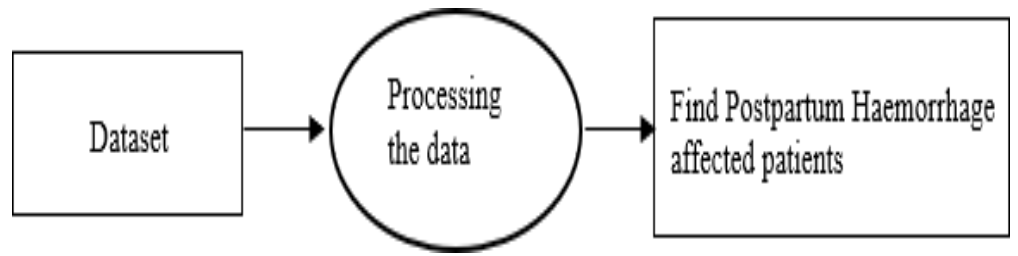

Figure 2. Level 0 DFD Diagram 


\subsection{Level 1}

By analysing the parameters, it will be easier to find the women who is affected by postpartum haemorrhage. If any changes occurs in the patient's body, it will be verified with the default value which has been stored previously in database. From the changes, it is easy to know whether the patient has reached critical level or not and it will give an intimation to doctor.

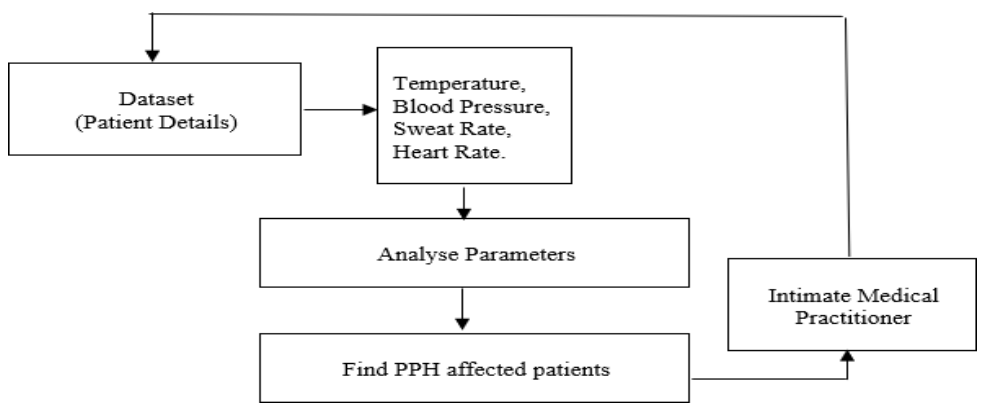

Figure 3. Level 1 DFD Diagram

\section{Conclusion}

In this paper, different parameters have been used and we can easily find the postpartum haemorrhage patients by analysing all the parameters. If the patient level goes abnormal, it makes sure to intimate the medical practitioner and the postpartum haemorrhage affected patients will be identified and prevented easily.

\section{References}

[1] Salamah, U., Kartikawangi, S., \& Suryani, E. Identification of Postpartum Infection Type using Mamdani Fuzzy System ,4th International Conference on Informatics and Computing, ICIC,2019.

[2] Michelle Kominiarek,A., MD, Sarah Kilpatrick, J., MD, PhD. Postpartum Haemorrhage: A Recurring Pregnancy Complication. Seminar in perinatology,2007.

[3] Zeni Montenegro, J. L., Andre Da Costa, C., Da Rosa Righi, R., Roehrs, A., \& Farias, E. R. A proposal for postpartum support based on natural language generation model , International Conference on Computational Science and Computational Intelligence, CSCI, 756-759,2018.

[4] Gowri Ramanathan, Sabaratnam Arulkumaran., Postpartum Haemorrhage, JOCG, 28, 11, 967-973 , 2006.

[5] Rancogz, Management of Postpartum Haemorrhage (PPH), Management of Postpartum Haemorrhage C-Obstetrics,(43), 2011.

[6] Radite Purwahana, R., Suryono, S., \& Suseno, J. E.. Cloud-Based Multinomial Logistic Regression for Analyzing Maternal Mortality Data in Postpartum Period ,2nd International Conference on Informatics and Computational Sciences, ICICOS, 13-16, 2019.

[7] Adegoke, O., Danso-Bamfo, S., Sheehy, M., Tarimo, V., Burke, T. F., \& Garg, L. F. A condom uterine balloon device among referral facilities in Dar Es Salaam: An assessment of perceptions, barriers and facilitators one year after implementation",BMC Pregnancy and Childbirth, 20(1), 16,2020 .

[8] Edwin Chandraharan, Archana Krishna, Diagnosis and management of postpartum haemorrhage, Diagnosis and management of postpartum haemorrhage. BMJ, j3875,2017.

[9] Brian Bateman, T., Md Mitchell Berman, F et al, The Epidemiology of Postpartum Haemorrhage in a Large, Nationwide Sample of Deliveries, Anesthesia \& Analgesi,110(5),1368-1373,2010. 
[10] Mousa HA, Blum J, Abou El Senoun G, Shakuretal, Treatment for primary postpartum haemorrhage, Cochrane Database of Systematic Reviews, Issue 2,2014.

[11] Dwight, J., Rouse, MD., MSPH ,What Is New In Postpartum Haemorrhage, American Journal of Obstetric and Gynecology,122(3),693-694,2013.

[12] Muhammad Muzzammil Edhi , Hafiz Muhammad Aslam, Zehra Naqvi, Edhi et al "Postpartum Haemorrhage : Causes And Management",BMC Research Notes, 6:236, 2013.

[13] Dean Leduc, MD., Ottawa Vyta Senikas, MD, Ottawa, ON., et al ,Active Management of the Third Stage of Labour: Prevention and Treatment of Postpartum Haemorrhage, Journal of Obstetrics and Gynaecology Canada, 31(10), 980-993,2009.

[14] Guillermo Carroli, Luciano Mignini. Episiotomy for vaginal birth. Cochrane Database of Systematic Reviews,(1),2009.

[15] Hema Kumar.S, J.Uday Kiran, V.D.AKumar, G.Saranya, Ramalakshmi V, "Effective Online Medical Appointment System", International Journal of Scientific \& Technology Research , Volume 8, Issue 09, September 2019, Pages 803-805.

[16] Ambeth Kumar.V.D, Dr.M.Ramakrishnan, V.D.Ashok Kumar and Dr.S.Malathi (2015) . Performance Improvement using an Automation System for Recognition of Multiple Parametric Features based on Human Footprint. for the International Journal of kuwait journal of science \& engineering, Vol 42, No 1 (2015), pp:109-132.

[17] Ambeth Kumar.V.D, V.D.Ashok Kumar, S.Malathi, K.Vengatesan, M.Ramakrishnan .Facial Recognition System For Suspect Identification Using A Surveillance Camera.Pattern Recognition and Image Analysis (Springer), Volume 28, Issue 3, pp 410-420, 2018. (DOI: $10.1134 /$ S1054661818030136)

[18] Ambeth Kumar.V.D and M.Ramakrishan .Employment Of Footprint Recognition System. in the month of December for Indian Journal of Computer Science and Engineering (IJCSE) Vol. 3 No.6 Dec 2013

[19] Ramya,T.,Dr.Malathi,S.,ratheeksha,G.R. and Dr.V.D.Ambeth Kumar. Personalized authentication procedure for restricted web service access in mobile phones.Applications of Digital Information and Web Technologies (ICADIWT), 2014, Page(s):69 - 74, (ISBN:978-1-4799-2258-1)

[20] Ambeth Kumar.V.D (2018). Novel Wireless Sensing System For The Welfare Of Sewer Laborers. Healthcare Technology Letters (IET) Volume 5, Issue 4, p. 107-112.

[21] V.D.Ambeth Kumar, G.Saranya , D.Elangovan, V.RahulChiranjeevi, V.D.Ashok Kumar, "IOT Based Smart Museum Using Wearable Device", Lecture Notes in Networks and Systems, Vol.55, pp: 33-42, 2018

[22] Ambeth Kumar.V.D and Dr.M.Ramakrishan(2011) .Footprint Based Recognition System. in the month of April for the International Journal Communication in Computer and Information System (CCIS) Journal (Springer) Volume 147, Part 3, 358-367, DOI: 10.1007/978-3-642-20573-6_63, April 2011

[23] Ambeth Kumar.V.D (2017).Efficient Routing for Low Rate Wireless Network a Novel Approach. International Journal of Image Mining, Vol. 2, Nos. 3/4, 2017, 2017

[24] Ambeth Kumar.V.D, V.D.Ashok Kumar, Dr.S.Malathi and P.Jagaeedesh, (2014) .Intruder Identification using Footprint Recognition with PCA and SVM Classifiers.for the International Journal of Advanced Materials Research Vols.1345, PP 984-985 (2014) pp 1345-1349. [DOI:10.4028/www.scientific.net/AMR.984-985.1345]

[25] Ambeth Kumar.V.D, G.Gokul, S.Malathi, K.Vengatesan, D.Elangovan, B.Chitra.Implementation Of The Pulse Rhythemic Rate For The Efficient Diagonising Of The Heart Beat. Healthcare Technology Letters (IET) 2019 Apr 17;6(2):48-52.

[26] Ruphitha.S.V et.al.Management of Major Postpartum Haemorrhage by using Zigbee protocol - A Review .2021 6th International Conference on Inventive Computation Technologies (ICICT) (DOI: 10.1109/ICICT50816.2021.9358757)

[27] Ambeth Kumar.V.F, Dr.M.Ramakrishnan, V.D.Ashok Kumar and Dr.S.Malathi .Performance Improvement using an Automation System for Recognition of Multiple Parametric Features based on Human Footprint. kuwait journal of science, Vol 42, No 1 (2015), pp:109-132.

[28] Ramakrishan.M et.al.,Footprint Based Recognition System.in the month of April for the International Journal Communication in Computer and Information System (CCIS), Volume 147, Part 3, 358367, DOI: 10.1007/978-3-642-20573-6_63, April 2011

[29] Indhumathi.M et.al .Healthcare Management of Major Cardiovascular Disease-A review. 2021 6th International Conference on Inventive Computation Technologies (ICICT), (DOI: 10.1109/ICICT50816.2021.9358519)

[30] Rajamani.V, S Murugavalli .A High Speed Parrallel Fuzzy C-means algorithm for Tumor Segmentation. ICGST International Journal on BIME 6 (1), 2006 